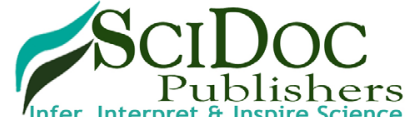

Publishers

\title{
HDFx: A Novel Immunomodulator and Potential Fighter Against Cytokine Storms in Inflammatory and Septic Conditions in Dogs and Farm Animals
}

Altura $\mathrm{BM}^{1-5^{*}}$, Gebrewold A ${ }^{1}$, Carella $\mathrm{A}^{1}$, Altura $\mathrm{BT}^{1,3-5}$

\begin{abstract}
${ }^{1}$ Department of Physiology and Pharmacology, The State University of New York Downstate Medical Center, Brooklyn, New York, USA.
${ }^{2}$ Department of Medicine, The State University of New York Downstate Medical Center, Brooklyn, New York, USA.

${ }^{3}$ Center for Cardiovascular and Muscle Research, The State University of New York Downstate Medical Center, Brooklyn, New York, USA.

${ }^{4}$ The School for Graduate Studies in Molecular and Cellular Science, The State University of New York Downstate Medical Center, Brooklyn, New York, USA.

${ }^{5}$ Bio-Defense Systems, Inc, Rockville Centre, New York, USA.
\end{abstract}

Over the past decade, a disturbing trend in antimicrobial resistance of both gram-negative and gram-positive "superbugs" in addition to fungal "superbugs" has seriously complicated the treatment of not only immune-compromised patients but dogs and farm animals (e.g., cattle, pigs, horses, etc) as well [1-8]. To this, must be added the numerous hospitalizations and veterinary visits caused by contaminated meats, poultry, vegetables, seafoods, and multiple types of animal feeds [9-12]. Government resources, worldwide, are being stretched-thin and often remain powerless to combat these assaults to our patients, dogs and farm animals. By about 2075, the number of people dying from drug-resistant infections and diseases could reach in excess of 35 million while the deaths in the pet and farm animal populations could be expected to far exceed this number. Added to this is the ever-growing and soaring worldwide use of antibiotics in agriculture. How much of this indiscriminate use of antibiotics is contributing to the ever growing resistance of pathogens to antibiotics noted above?

A major problem associated with infectious microorganisms and fungi is the underlying production of "cytokine storms (CS)" which often result in very severe illness and death. The major factors producing these CS usually are tissue trauma, burns, and blood loss which cause extensive tissue damage of large numbers of cells of diverse types, particularly those of the innate and adaptive immune systems. But, it should be kept in mind that multiple virus infections, such as dengue, hanta viruses, diverse hemorrhagic fever viruses, and influenza viruses can also trigger massive cell destruction, resulting in extensive cytokine release and death $[13,14]$.

Septic shock caused by severe bacterial infections accounts for about $10 \%$ of all human deaths in the U.S.A., alone, each year, and is a major cause of farm animal deaths each year. These severe bacterial infections in both humans and animals are associated with characteristic signs such as fevers, myalgias, rigor, depression, and nausea, resulting from release of cytokines. Major cytokines involved in these septic-inflammatory states are TNF-alpha, IL1 beta, IFN-gamma, IL-6, and IL-8 among others, as well as several macrophage factors. These cytokines and chemokines when released then go-on to cause increases in nitric oxide synthases, leading to nitric oxide and COX-2 which then result in release of diverse tissue-damaging prostanoids and leukotrienes, production of severe acidosis, fever, tissue lactate release, uncontrollable falls in arterial blood pressure, elevations in plasma histamine, serotonin, kinins and catecholamines, eventuating in multiple organ failure (particularly of the kidneys, lungs, and heart). This sequelae of events prior to death usually causes intravascular coagulation and vast increases in capillary permeability leading to extensive fluid loss $[15,16]$. The predilection of various animals to sepsis varies greatly [15]. Those species with pulmonary intravascular macrophages (i.e., cats, horses, sheep, and pigs) are more susceptible than dogs which lack pulmonary intravascular macrophages and are much less susceptible to lung injuries [15].

Our laboratories have been working on a brand-new approach to develop host-defense factors that stimulate various arms of the innate and adaptive immune systems. To this end, we have discovered a new host-defense factor, termed "HDFx", that is a conserved $35-40 \mathrm{kD}$ protein found in mice, rats, gunea-pigs, rabbits, dogs, cats, piglets, and sub-human primates [16-22]. We assume it is also present in humans and farm animals such as sheep, cattle, pigs, and horses, particularly since it is a conserved molecule. More than 135 years ago, Elie Metchnikoff, the great father of immunology, hypothesized that the body, under stressful circumstances, might produce powerful immunostimulants which perforce would act on different arms of the innate immune system and serve to protect

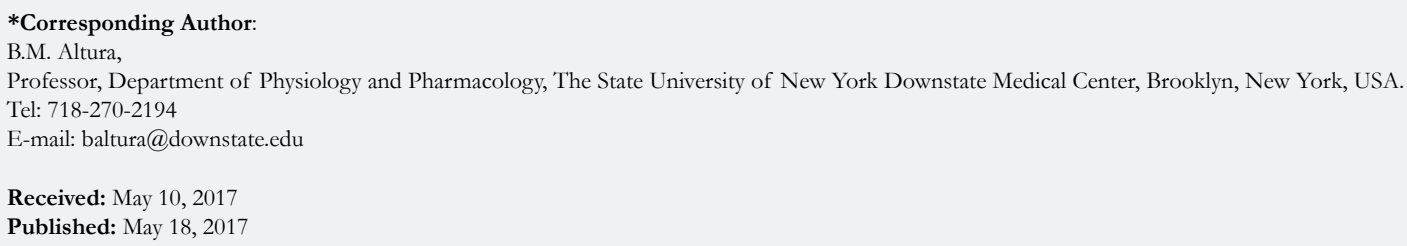

Copyright: Altura $\mathbf{B M}^{\circ}$ 2017. This is an open-access article distributed under the terms of the Creative Commons Attribution License, which permits unrestricted use, distri bution and reproduction in any medium, provided the original author and source are credited. 
against major insults, inflammatory conditions, and diseases [23]. Metchnikoff's early studies pointed to the important contributions of macrophages and phagocytic leukocytes to natural (innate) resistance against pathogenic bacteria and viruses. Over the past 40 years, considerable evidence has accumulated to support a strong relationship between the functional (physiological) state of the microcirculation, macrophages-phagocytes, natural killer (NK) cells, the reticuloendothelial system, and "pit cells" in the liver to host defense and resistance to pathogens, trauma, sepsis, wounding, circulatory shock, and combined injuries [4, 5, 24-31].

Recent studies from our laboratories have clearly shown that HDFx is protective (to different degrees) against a variety of systemic insults ranging from hemorrhage, trauma, endotoxins, a variety of bacteria (e.g., E. coli, S. enteriditis, C. wechii, among others), combined injuries, and centripetal forces to septic shock [1622]. A unique attribute of HDFx is that it can accelerate wound healing [21]. Most importantly, HDFx has been demonstrated in several animal models to inhibit the release of multiple cytokines and chemokines, including TNF-alpha, IL-6. IL-8, Il-1beta, IFNgamma, numerous and macrophage factors [18-22]. In other terms, HDFx clearly either prevents or ameliorates the intensity of "cytokine storms" induced by both gram-negative and grampositive bacteria, toxic fungal microorganisms, trauma, systemic inflammatory conditions, tissue damage, blood loss, and sepsis, among other dangerous insults [[16-22], unpublished findings]. HDFx also has protective qualities even in diseases such as nonalcoholic steatohepatitis (NASH) which often results in liver carcinomas [20]. We have also produced preliminary data to suggest that HDFx might be useful in the treatment and amelioration of hemorrhagic fevers [22].

Gram-negative "superbugs" seem to be the major culprits in many hospitalized patients, very sick dogs, and a variety of sickly farm animals. Gram-negative bacteria appear to be more difficult to kill than gram-positive bacteria because they are protected by "double membranes". So, in order to kill the gram-negative bacteria, most of the approaches have been to design antibiotics to penetrate these membrane barriers. In our opinion, another likely approach would be to engulf the bacteria and digest them within "supercharged" macrophages, Kupffer cells, phagocytic leukocytes, platelets, "pit cells" and NK cells. HDFx appears, at least experimentally, to induce a "supercharged effect" in these various cell types in all animals we have investigated to date. But, for this to occur, in an expeditious manner, we believe the microcirculation to key organs and tissues must perforce produce optimal capillary blood flows and distribution. Therefore, an ideal drug or therapeutic molecule would be one that could stimulate multiple arms of the innate immune system coupled to modulation of microcirculatory blood flows to the aforementioned key organ and tissue systems. So far, of all molecules, we have investigated, HDFx appears to be the only molecule that embodies all these qualities and demonstrates therapeutic attributes against several classes of "superbugs" [16-22].

We, thus, believe that the approaches outlined in the above, using HDFx or its derivatives, could be the ideal drug (s) to pretreat all patients scheduled for major surgeries as well as all cats, dogs and farm animals to prevent a variety of infections and assaults from "superbugs".

A major objective of our group is to secure adequate funding to elucidate the complete, complex molecular structure of HDFX and then via genetic engineering to produce large quantities of HDFx for further testing in human subjects and farm animals under diverse pathophysiological conditions, including infections induced by "superbugs".

\section{Acknowledgements}

Some of the original studies and thoughts needed for the discovery of HDFx and reviewed, above, were initiated while the first author was at New York University School of Medicine and The Albert Einstein College of Medicine of Yeshiva University. Some of the original studies reviewed, above, were supported, in part, by unrestricted research grants from several pharmaceutical companies (CIBA-GEIGY Pharmaceuticals, The UpJohn Company, Sandoz Pharmaceuticals, and Bayer Pharmaceuticals) and anonymous donors. The authors are indebted to many colleagues, over many years, who helped to make our studies and background experiments possible: C.Thaw, E.W. Burton, J. Hanley, and C. Parillo. The authors are also grateful to Dr. Solomon G. Hershey (deceased) who suggested several animal models to us.

\section{References}

[1]. Gaynes E, Edwards R (2005) Overview of nosocomial infections caused by gram-negative bacilli. Clin Infect Dis. 41(6): 948-954.

[2]. Blossom DB, McDonald LC (2007) The challenges posed by reemerging Clostridium difficile infection. Clin Infect Dis. 45(2): 222-227.

[3]. Burton DC, Edwards JR, Horan TC, Jernigan JA, Fridkin SK (2009) Methicillin - resistant Staphylococcus aureus central line-associated bloodstream infections in US intensive care units. JAMA. 301(7): 727-736.

[4]. Lee JH, Jeong SH, Cha SS, Lee SH (2009) New disturbing trend in antimicrobial resistance of gram-negative pathogens. PloS Pathog. 5(3): e1000221.

[5]. Kuehn BM (2007) Antibiotic-resistant "Superbugs" may be transmitted from animals to humans. JAMA. 298(18): 2125-2126.

[6]. Holden MTG, Hauser H, Sanders M, Ngo TH, Cheverach I, et al., (2009) Rapid evolution of virulence and drug resistance in emerging pathogen Streptococcus suis. PloS One. 4(7): e6072.

[7]. Marston HD, Dixon DM, Knisely JM, Palmore TN, Fauci AS (2016) Antimicrobial resistance. JAMA. 316(11): 1193-1124.

[8]. Zignol M, Dean AS, Falzom D, van Gemert W, Wright A, et al., (2016) Twenty years of global surveillance of antituberculosis-drug resistance. $\mathrm{N}$ Engl J Med. 375(11): 1081-1089.

[9]. Anonymous (2009) Preliminary foodnet data on the incidence of infections with pathogens transmitted commonly through food-10 states,2008. MMWR. 158(13): 333-337

[10]. Maki DG (2009) Coming to grips with foodborne infection- Peanut, butter, pepper, and Nationwide Salmonella outbreaks. N Engl J Med. 360(10): 949-953.

[11]. Schiller LR (2009) Infectious disease: A germy world-foodborne infections in 2009. Nature Rev Gastroenterol Hepatol. 6(4): 197-198.

[12]. Tierno PM (2001) The Secret Life of Germs: Observations and Lessons from a Microbe Hunter. Pocket Books, New York.

[13]. Majno G, Joris I (2004) Cells, Tissues and Diseases. (2nd edn), Oxford University Press, New York.

[14]. Murphy K, Weaver C (2016) Janeway’s Immunology. (9th edn), Garland Science, New York.

[15]. Constable PD, Hinchcliff KW, Done SH, Grunberg W (2017) Veterinary Medicine, vil I. (11th edn), Elsevier, St Louis.

[16]. Altura BM (2016) HDFx: A novel immunomodulator for the amelioration of hypovolemic shock in the OR, cancer patients and on the battlefield. J Clin Med Therap. 1(1): e003.

[17]. Altura BM, Gebrewold A, Carella A (2009) A novel biologic immunomodulator, $\mathrm{HDF}$, protects against lethal hemorrhage, endotoxins and traumatic injury: potential relevance to emerging diseases. Int J Clin Exp Med. 2(3): 266-279.

[18]. Altura BM, Gebrewold A, Carella A (2011) HDFx: a novel biologic immunomodulator is therapeutically-effective in hemorrhagic and intestinal ischemic shock: importance of microcirculatory-immunological interactions and their implications for the warfighter and disaster victims. Int J Clin Exp Med. 4(4): 331-340. 
[19]. Altura BM, Carella A, Gebrewold A (2012) HDFx: a novel biologic immunomodular accelerates wound healing and is suggestive of unique regenerative powers: potential implications for the warfighter and disaster victims. Int J Clin Exp Med. 5(4): 289-295.

[20]. Altura BM, Gebrewold A, Carella A, Altura BT (2016) HDFX: A potential new treatment and prophylactic against nonalcoholic steatohepatitis (NASH) and subsequent hepatocellular carcinomas: Is hypomagnesemia a complication of the disease? J Alcoholism Drug Depend. 4: 10000e133.

[21]. Altura BM (2016) HDFx: A novel immunomodular and potential superbug super- warrior for hospitalized patients and battlefield casualties. Int J Vaccines and Res. 3: 1-3.

[22]. Altura BM, Gebrewold A, Carella (2016) HDFx: A recently discovered biologic and its potential use in prevention and treatment of hemorrhagic fever viruses and antibiotic-resistant superbugs. J Hematol Thromboembolic Dis. 4: 1000252.

[23]. Metchnikoff E (1884) Untersuchung ueber die intracellular Verdauung beiwirbellosen Thieren. Arbeiten aus dem Zoologischesn Institut zu Wien. 5(2): $141-168$.

[24]. Altura BM, Hershey SG (1968) RES phagocytic function in trauma and adaptation to experimental shock. Am J Physiol. 215: 1414-1419.
[25]. Hershey SG, Altura BM (1969) Function of the reticuloendothelial system in experimental shock and combined injury. Anesthesiology. 30(2): 138-143.

[26]. Altura BM, Hershey SG (1970) Effects of glycerol trioleate on the reticuloendothelial system and survival after rxperimental shock. J Pharmacol Exp Ther. 175(3): 555-564.

[27]. Altura BM, Hershey SG (1972) Sequential changes in reticuloendothelial function after acute hemorrhage. Proc Soc Exp Biol Med. 139(3): 935-939.

[28]. Altura BM (1974) Hemorrhagic shock and reticuloendothelial system phagocytic function in pathogen-free animals. Circulatory Shock. 1: 295300.

[29]. Altura BM, Gebrewold A (1980) Prophylactic administration of antibiotics compromises reticuloendothelial system function and exacerbates shock mortality in rats. Br J Pharmacol. 68(1): 19-21.

[30]. Altura BM (1982) Reticuloendothelial system function and histamine release in shock and trauma: relationship to microcirculation. Klin Wochenschr. 60(17): 1021-1030.

[31]. Altura BM (1985) Microciculatory regulation and dysfunction: Relation to RES function and resistance to shock and trauma. The Reticuloendothelial System. Plenum Press, New York. 7: 353-395. 